\title{
A Comparative Analysis of Performance Evaluation of SVC and TCSC for Transmission Loss Reduction on Nigerian 330 kV Grid System Using Self- Adaptive Firefly Algorithm
}

\author{
Ganiyu Adedayo Ajenikoko, Olakunle Elijah Olabode, George Ojo
}

Department of Electronic \& Electrical Engineering, Ladoke Akintola University of Technology, P.M.B. 4000, Ogbomoso, Nigeria

Corresponding Email: ajeedollar@gmail.com

Received: 25.11.2017 Accepted:30.12.2017

\begin{abstract}
Flexible alternating current transmission systems (FACTS) devices are emerging technologies that serve as a means of providing reactive power support to mitigate issues of active power loss and voltage instability on existing power grid. The optimal placement, locations and sizes of these devices influence its performance on the grid. This paper presents a comparative analysis of performance evaluation of SVC and TCSC for transmission loss reduction on Nigerian 330kV grid system using self- adaptive firefly algorithm. The results of the analysis showed that with the system reinforced with SVC, the total system loss reduced from 98.21MW to $92.44 \mathrm{MW}$ which is about $5.88 \%$ reduction. The reduction in active power loss with the optimal location of TCSCs is 94.259MW which amount to $4.02 \%$ reduction. An appreciable voltage enhancement occurred at Ayede, New Haven, Onitsha and Kano due to the system reinforcement with SVCs and TCSCs. In all, SVC gives better result than TCSC in term of active power reduction and voltage profile enhancement.
\end{abstract}

Keywords: Active Power Loss, FACTS, Optimal Location, Self-Adaptive Firefly Algorithm, SVC, TCSC, Transmission Loss Reduction, Voltage Profile Enhancement,

\section{Introduction}

The exponential increase in electrical power demand alongside long distance transmission of energy from the generating end to load centers coupled with enormous and complex power transmission lines poses threat to the power network stability and this is making the interconnected systems increasingly difficult to operate reliably and efficiently [[18], [12]]. This scenario creates a growing need for flexibility, reliability, fast response and accuracy of power system in a bid to addressing this problem [12]. There are several means of mitigating this problem; such methods include installation of new transmission networks and new generating power plants. Unfortunately, these methods are associated with the following setbacks; expensive initial installation cost, degradation of environment and bottlenecking in the land acquisition [9].

The advent of modern power electronics technology led to the development of FACTS device which offered the simplest way for minimizing real power loss on power system [8]. FACTS devices are used in increasing system transmission capacity, system security and power flow control by means of efficient management flow of the reactive power in electric power systems [[15], [3]]. FACTS controllers are classified as Series (Thyristor Controlled Series Compensator (TCSC) and Static Synchronous Series 
Compensator (SSSC), Shunt (Static VAR Compensator (SVC) and Static Synchronous Compensator (STATCOM)) and combined Series-Shunt Unified power flow controller (UPFC) devices based on their existence in the system [[8], [11]].

Transmission loss minimization through FACTS devices is an optimization problem that has been addressed by a number of researchers using both conventional and evolutionary algorithms. Newton Raphson technique has been proposed for transmission loss minimization incorporating TCSC and SVC devices [13]. Genetic Algorithm (GA) has been implemented for voltage stability enhancement using SVC and TCSC [1]. Particle Swarm Optimization (PSO) technique for voltage stability enhancement and power loss minimization using SVC has been implemented successfully [18]. Bees algorithm was implemented for optimal allocation of FACTS devices for automatic transmission controller enhancement [10] and Self-Adaptive Firefly algorithm (SAFA) has also been implemented using both TCSC and SVC for voltage constrained and transmission loss minimization on IEEE bus system [12].

FACTS devices are installed at different locations of the test case power system network, the depth of loss minimized and the extent of voltage profile enhancement were used as the performance metric. One-line diagram of the Nigerian $330 \mathrm{kV}, 28$-bus system interconnecting nine generator buses, nineteen load buses and fifty-two transmission lines is shown in Figure 1

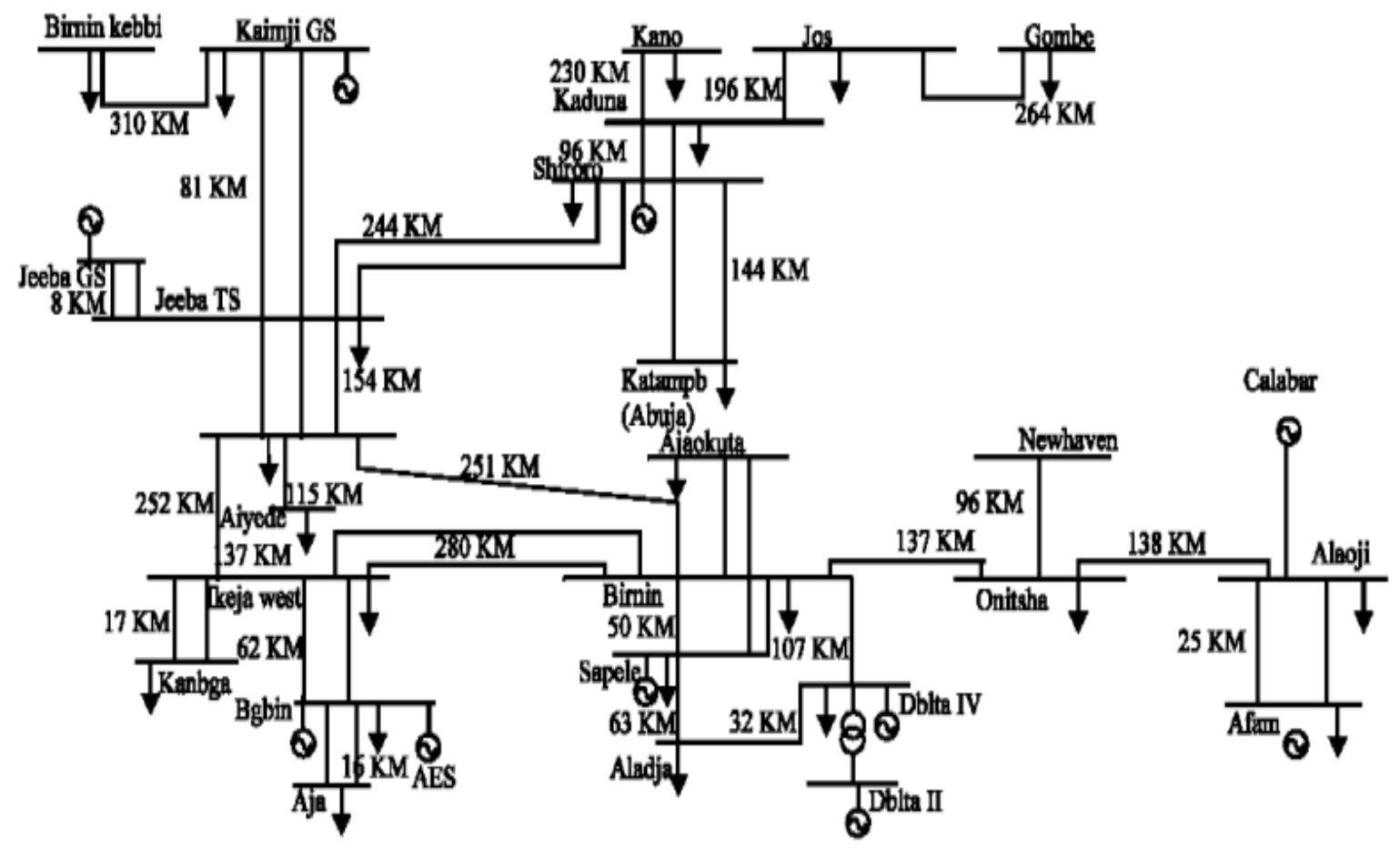

Figure 1: 28-bus, 330kV Nigerian Transmission System (Source: National Control Centre, Osogbo, PHNC, 2009)

\section{Concept of Self-Adaptive Firefly Algorithm}

Nature-inspired metaheuristic algorithms are used in solving complex engineering optimization problems. One of the recent swarm-intelligence (SI) optimization techniques belonging to this group is Firefly Algorithm (FA) developed by Yang in 2007 [20]. FA produces a swarm of fireflies which are located stochastically in the search space and this allows for thorough exploration and exploitation in addition to the fact that it works on global communication among swarming particles (Fireflies).

Firefly Algorithm since its inception has caught the attention of dynamic researchers and it has been used to solve various engineering problems such as convex and nonconvex economic load dispatch [[2], [14]], job scheduling [21], fault identification [7] and travelling salesman problem [5]. Firefly has some basic control parameters that are crucial if the algorithm will converge to global optimum. These 
basic control parameters are the random movement factor $(\alpha)$, the attractiveness $(\beta)$ and the absorption coefficient $(\gamma)$. Improper choice of these parameters will automatically affect the optimal solution and as a result the algorithm may converge at local optimal.

The original Firefly algorithm is associated with this problem since control parameter values cannot be modified during each iteration process. One way to guide against this problem is by modifying the control parameter values in each stage of iteration run and this variant of firefly algorithm is called Self-Adaptive Firefly (SAFA). SAFA is endowed with the ability to achieve better convergence in addition to obtaining global best solution since these parameters $\alpha, \beta$ and $\gamma$ are tuned through a self-adaptive mechanism at each iteration [17].

\section{Materials and Method}

\subsection{Mathematical Model of Static Var Compensator (SVC)}

SVC has the ability to maintain an acceptable voltage level at any desired bus, they are shunt connected either as static VAR generator to raise the voltage level or static VAR absorber to decrease the voltage level within the permissible limit. The equivalent circuit of variable susceptance model of SVC is as shown below in Figure 2

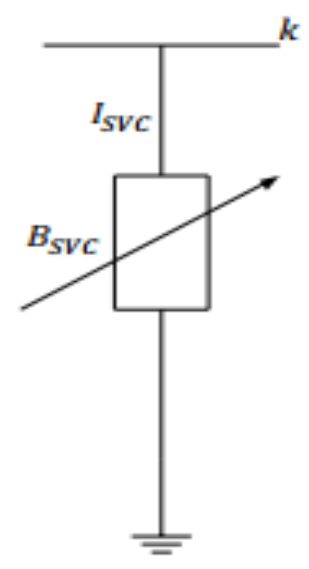

Figure 2: Variable susceptance model of SVC

Current drawn by the SVC is given by equation;

$I_{S V C}=j B_{S V C} V_{i}$

The reactive power injected by SVC at bus $i$ is given as;

$Q_{S V C}=Q_{i}=-V_{i}^{2} B_{S V C}$

The linearized equation representing the total susceptance $B_{S V C}$ as state variable is given as;
$\left[\begin{array}{c}\Delta P_{i} \\ \Delta Q_{i}\end{array}\right]^{k}=\left[\begin{array}{cc}0 & 0 \\ 0 & \frac{\partial Q_{i}}{\partial B_{S V C}}\end{array}\right]^{k}\left[\begin{array}{c}\Delta \theta_{i} \\ \Delta B_{S V C}\end{array}\right]^{k}$

At each iteration $(k)$, the variable shunt susceptance $B_{S V C}$ is updated as;

$B_{S V C}^{k+1}=B_{S V C}^{k}+\Delta B_{S V C}^{k}$

\subsection{Mathematical Model of Thyristor Controlled Compensator (TCSC)}

TCSC has the ability to control the active power flow in a line by varying the line reactance; they are connected in series with the transmission line to compensate the inductive reactance. It reactance depends on its compensation ratio and the reactance of the transmission line where it is cited. The model of TCSC is as shown below in Figure 3;

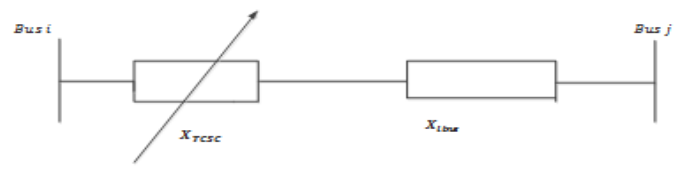

Figure 3: TCSC model

The TCSC modelled by the reactance $X_{\text {TCSC }}$ is expressed as follows;

$X_{i j}=X_{\text {line }}+X_{T C S C}$

$X_{\text {TCSC }}=\gamma_{\text {TCSC }} X_{\text {line }}$

The variable series compensator expressed in transfer admittance matrix form is as follows;

$\left[\begin{array}{l}\Delta I_{i} \\ \Delta I_{j}\end{array}\right]=\left[\begin{array}{ll}j B_{i i} & j B_{i j} \\ j B_{j i} & j B_{j j}\end{array}\right]\left[\begin{array}{l}V_{i} \\ V_{j}\end{array}\right]$

For inductive operation we have;

$B_{i i}=B_{j j}=-\frac{1}{X_{T C S C}}$

$B_{i j}=B_{j i}=\frac{1}{X_{T C S C}}$

The incremental change in the reactance is given as;

$\Delta X_{T C S C}=X_{T C S C}^{(i)}-X_{T C S C}^{(i-1)}$

At each iteration run, the reactance $\left(X_{T C S C}\right)$ is updated thus;

$X_{T C S C}^{(i)}=X_{T C S C}^{(i-1)}+\left(\frac{\Delta X_{T C S C}}{X_{T C S C}}\right)^{(i)}(X)_{T C S C}^{(i-1)}$ 


\subsection{Mathematical Model of Self-Adaptive Firefly Algorithm}

The detailed mathematical formulation for SelfAdaptive Firefly Algorithm is reported in [12] and is adopted for this work. An $m^{\text {th }}$ firefly in the conventional firefly algorithm is donated with a vector $x_{m}$ given as;

$x_{m}=\left[x_{m}^{1}, x_{m}^{2}, x_{m, \ldots}^{3}, x_{m}^{n d}\right]$

For SAFA, a firefly is represented by adding three decision variables into equation (12), thus transforming it into;

$x_{m}=\left[x_{m}^{1}, x_{m}^{2}, x_{m, \ldots,}^{3} x_{m}^{n d}, \alpha_{m}, \beta_{m i n, m}, \gamma_{m}\right]$

The limitation on the search space is imposed by the inequality constraints defined as;

$x^{v}(\min ) \leq x^{v} \leq x^{v}(\max ) \ldots v=1,2,3 \ldots ., n d$

The positions of fireflies are generated using uniform distribution equation given as;

$x_{m}^{v}=x^{v}(\min )+\left(x^{v}(\min )-x^{v}(\max )\right) \times$ rand

The light intensity of $m^{\text {th }}$ firefly, donated as $I_{m}$ given as; $I_{m}=\operatorname{Fitness}\left(x_{m}\right)$

The attractiveness between $m^{\text {th }}$ and $n^{\text {th }}$ Firefly $\beta_{m, n}$ is defined as;

$\beta_{m, n}=\left(\beta_{\max , m, n}-\beta_{\min , m, n}\right) e^{\left(-\gamma_{m r_{m}^{2}, n}\right)}+\beta_{\min , m, n}$

Where $\mathrm{r}$ is the distance between $m^{\text {th }}$ and $n^{\text {th }}$ firefly given as;

$r_{m, n=}\left|x_{m}-x_{n}\right|=\sqrt{\sum_{v=1}^{d}\left(x_{m, v}-x_{n, v}\right)^{2}}$

If the light intensity of $n^{\text {th }}$ firefly is comparatively brighter than that of $m^{\text {th }}$ firefly, the $m^{\text {th }}$ firefly is attracted towards $n^{\text {th }}$ firefly, and its motion is defined as; $x_{m}(k)=x_{m}(k-1)+\beta_{m, n}\left(x_{m}(k-1)-x_{m}(k-1)\right)+$ $\alpha($ rand -0.5$)$

\subsection{Problem Formulation}

The test case system used for this analysis is independently reinforced with SVC and TCSC. The SVCs and TCSCs are installed at appropriate locations within the test case system using SAFA with the aim of minimizing the real power losses and raising the voltage at defective buses within the acceptable range of 0.95 p.u to 1.05 p.u without any special attention on the installation cost.

\subsubsection{Objective Function}

The mathematical model that minimizes real power loss is defined as;

$\operatorname{Min} P_{\text {loss }}=\sum_{i=1}^{n} G_{i}\left(V_{i}^{2}+V_{j}^{2}-2 V_{i} V_{j} \cos \delta_{i, j}\right)$

\subsubsection{System Constraints}

The two fundamental constraints on power system are equality constraint (power balanced constraint) and the inequality constraint.

The equality constraints are the power balanced equations defined thus;

$P_{G i}-P_{D i}=P_{i}(V, \delta)$

$Q_{G i}-Q_{D i}=Q_{i}(V, \delta)$

The inequality constraints are the limitation imposed on the system which includes voltage limits, reactive power limit, SVC and TCSC limits. These limits are defined by the following equations;

Voltage constraints on the generator (PQ) - bus is given by equation (24);

$V_{\min }^{i} \leq V_{i} \leq V_{\max }^{i}$

The reactive power generation limit on the load (PV)-bus is thus;

$Q_{G i}^{\min } \leq Q_{G i} \leq Q_{G i}^{\max }$

The SVC rating constraint is defined thus;

$-100 M V A R \leq Q_{S V C} \leq 100 M V A R$ for $\mathrm{SVC}$

The TCSC rating constraint is given as;

$-0.8 X_{\text {line }} \leq X_{\text {TCSC }} \leq 0.2 X_{\text {line }}$ p. $u$

The SAFA based optimal location of SVC problem is given as;

$x_{m}=$
$\left\{\begin{array}{c}\left(L_{i}, Q_{S V C, 1} \alpha_{m,} \beta_{\min , m,} \gamma_{m}\right) \ldots\left(L_{m}, Q_{S V C M}, \alpha_{m}, \beta_{\min , m}, \gamma_{m}\right) \ldots \\ \left(L_{N}, Q_{S V C N}, \alpha_{N}, \beta_{\min , N}, \gamma_{N}\right)\end{array}\right\}$

The SAFA based optimal location of TCSC problem is given as;

$$
\begin{aligned}
& x_{m}= \\
& \left\{\begin{array}{c}
\left(L_{i}, \gamma_{T C S C, 1} \alpha_{m}, \beta_{\min , m,} \gamma_{m}\right) \ldots\left(L_{m,} \gamma_{T C S C, m}, \alpha_{m}, \beta_{m i n, m,}, \gamma_{m}\right) \ldots \\
\left(L_{N,}, \gamma_{T C S C, N}, \alpha_{N}, \beta_{\min , N}, \gamma_{N}\right)
\end{array}\right\}
\end{aligned}
$$

SAFA finds optimal solution by minimizing the light intensity and the light intensity is obtained by 
transformation of power loss function into intensity function defined by;

$$
\text { Maximized } I_{\max }=\frac{1}{1+\left(P_{\text {loss }}+\sum_{i \in \varphi}\left(V_{i}-V_{i}^{\text {limit }}\right)^{2}\right)}
$$

Simulation was carried out in MATLAB programming language with appropriate range of limits for both the control and dependent variable used for SAFA. The population size and maximum iteration were 50 and 100 respectively.

\section{Result and Discussion}

The results of the power system variable and SAFA parameters limit in terms of the maximum and minimum values are shown in Figure 4. The minimum and maximum values of the voltage magnitudes are 0.950 and 1.500 p.u respectively while the minimum and maximum reactive powers for SVC are -100MVAR and 100MVAR respectively. In this case, the minimum MVA reactance of the TCSC is -0.8 and 0.2 . The randomness, attractiveness and absorption of SAFA have minimum and maximum values of 0.0 and $0.6,0.4$ and 1.0 as well as 0.0 and 1.0 respectively.

Figure 5 illustrates the optimal location and parameters of SVCs for the SAFA. Ayede which is bus number 9 has a reactive power of $9.871 \mathrm{MVAR}$ while New Haven, bus number 13 has a reactive power of 6.021MVAR, Onitsha, bus 14 and Kano, bus 22 have reactive powers of 11.101MVAR and 11.606 MVAR respectively. Figure 6 shows the optimal location and parameters of TCSCs for the SAFA. Between lines 5-9, there is an admittance of 0.200 p.u. In a similar manner, lines 13-14, 12-14 and 20-23 have TCSC admittance of 0.187 p.u, 0.209 p.u and 0.141 p.u respectively.

With the test case system reinforced with SVCs and TCSCs using SAFA techniques for optimal placement of these FACTs devices, the resultant voltage profile enhancement on the system is illustrated in Figure 7, Ayede, New- haven, Onitsha and Kano have base voltage magnitudes of 0.972 p.u, 0.946 p.u , 0.967 p.u and 0.963 p.u respectively. With the incorporation of SVCs, the voltage magnitudes for Ayede, New-haven, Onitsha and Kano are 1.024 p.u, 1.00p.u, 1.00p.u and 1.050 p.u while with TCSCs, the voltage magnitudes for Ayede, New-haven, Onitsha and Kano are 1.011 p.u, 0.989 p.u 0.996 and 1.011 p.u respectively.
With the incorporation of SVCs, the voltage magnitudes improved appreciably compared to when TCSCs were incorporated. Thus, the voltage magnitudes in Ayede, New-Haven, Onitsha and Kano are 1.011 p.u, 0.989 p.u, 0.996 p.u and 1.013 p.u respectively, thus confirming the efficiency of SVCs that had voltage magnitudes of 1.024 p.u, 1.00 p.u. 1.00 p.u and 1.050 p.u respectively. Figure 8 illustrates the percentage voltage profile enhancement on the test case system with the system independently reinforced with SVCs and TCSCs. The percentage increase in voltage profile with SVCs for Ayede, New-Haven, Onitsha and Kano are $5.34 \%, 3.41 \%, 3.41 \%$ and $10.79 \%$ respectively, thus confirming the efficiency of the SVCs. The TCSC is less efficient with percentage increase of $4.01 \%, 2.99 \%$, $2.99 \%$ and $5.06 \%$ for Ayede, New-Haven, Onitsha and Kano respectively.

The effect of the system reinforced with SVCs and TCSCs bring about an appreciable reduction in the active power loss of the system as shown in Figure 9. The active power losses with SVCs and TCSCs are 92.44MW and 94.259 MW respectively with a base case of 98.21MW. This represents a reduction in active power loss of 5.744MW and 3.951 MW with SVCs and TCSCs respectively and a percentage reduction in active power losses of $5.8 \%$ and $4.02 \%$ with SVCs and TCSCs respectively.

A comparison of the voltage magnitudes with SVC and TCSC is depicted in Figure 10, it shows the voltage profile enhancement capabilities of SVC and TCSC with the SAFA. Figure 11 shows the comparison of the increase in active power losses for the base case with SVC and TCSC. The decrease in the active power loss as well as the percentage decrease in active power loss is also shown in Figure 12. The reduction in active power loss with the optimal location of TCSCs is $94.259 \mathrm{MW}$ amounting to $4.02 \%$ reduction.

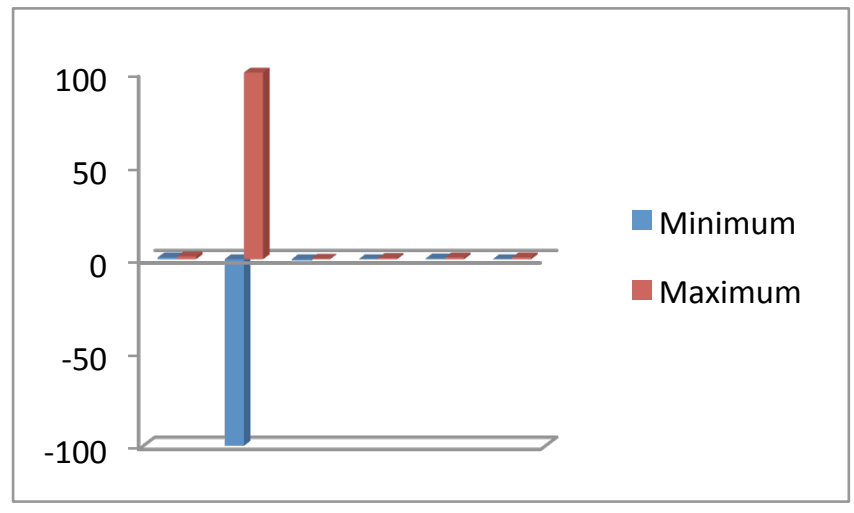

Figure 4: The graph showing the power system and SAFA parameters limits 


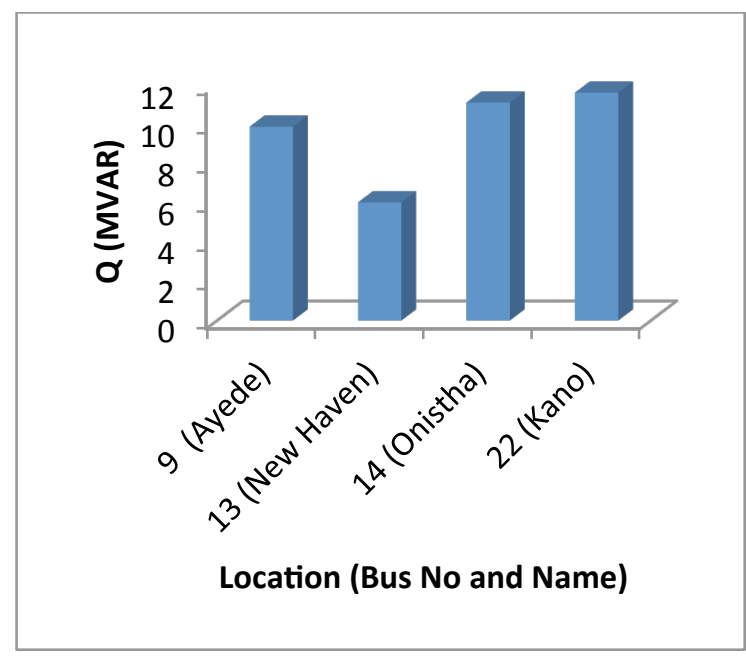

Figure 5: A graph showing the optimal location and parameters of SVCs

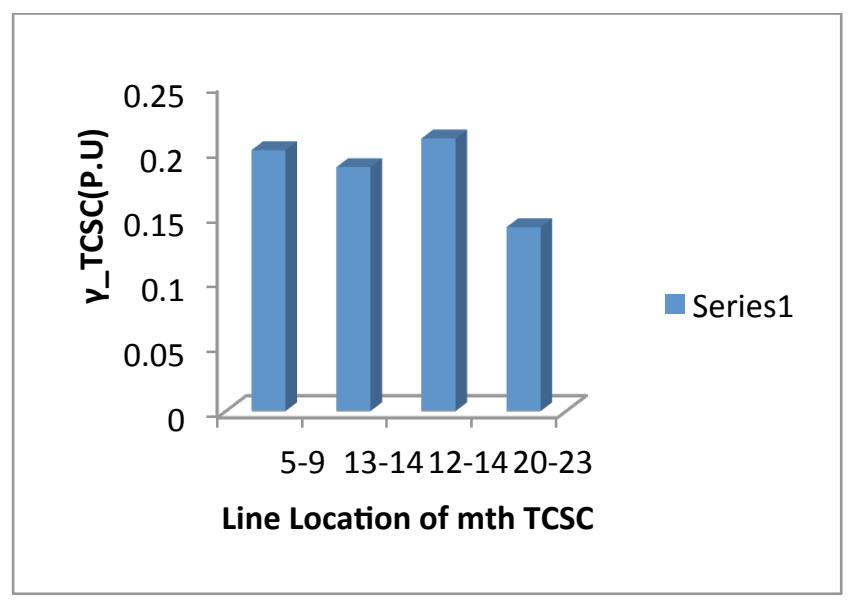

Figure 6: A graph showing the optimal location and parameters of TCSCs

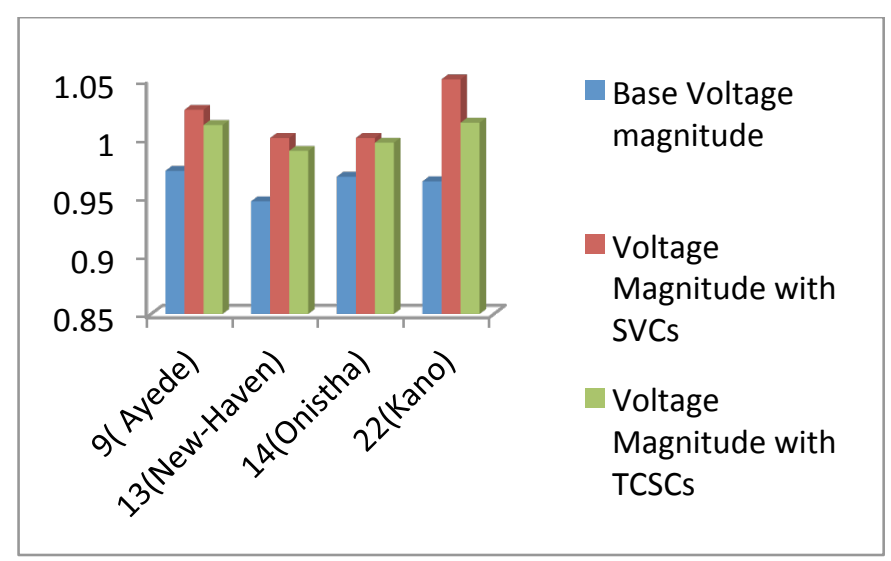

Figure 7; A graph showing voltage magnitude comparison of TCSC and SVC

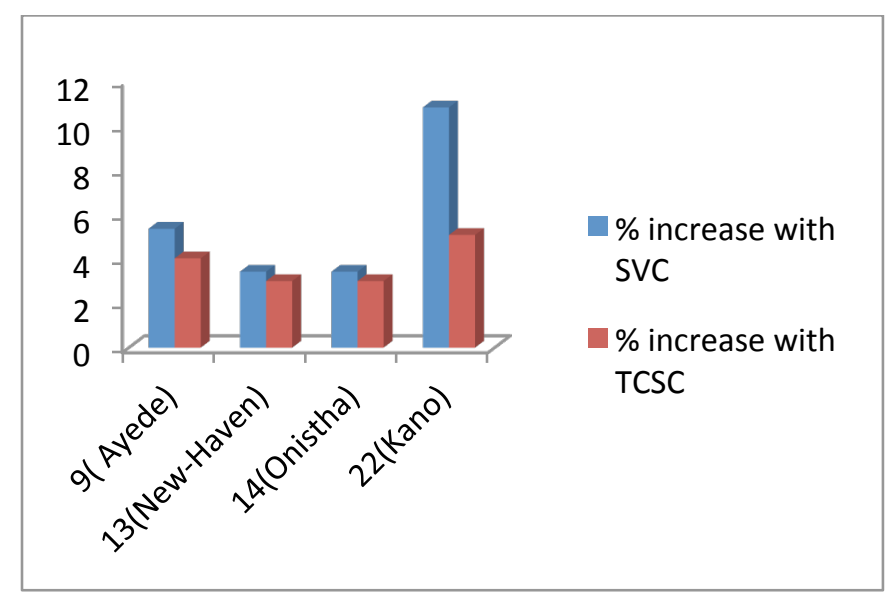

Figure 8: A graph showing percentage improvement comparison of TCSC and SVC

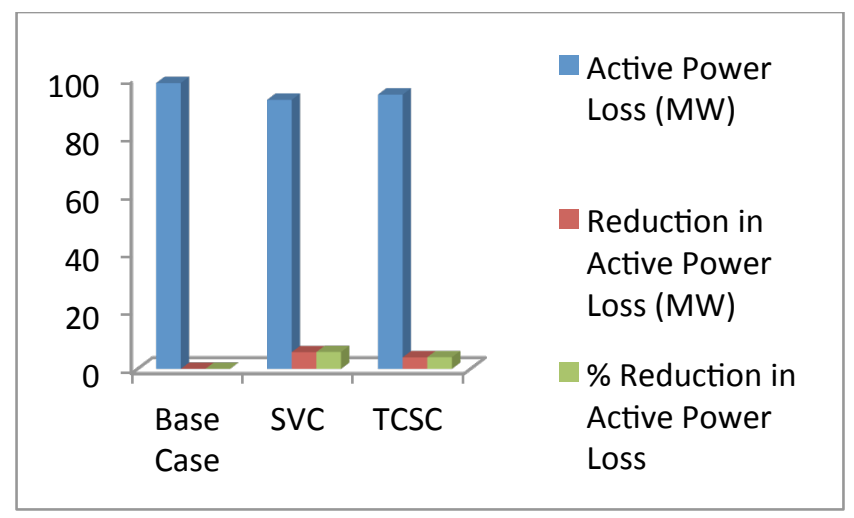

Figure 9: A graph of active power loss with TCSC and SVC

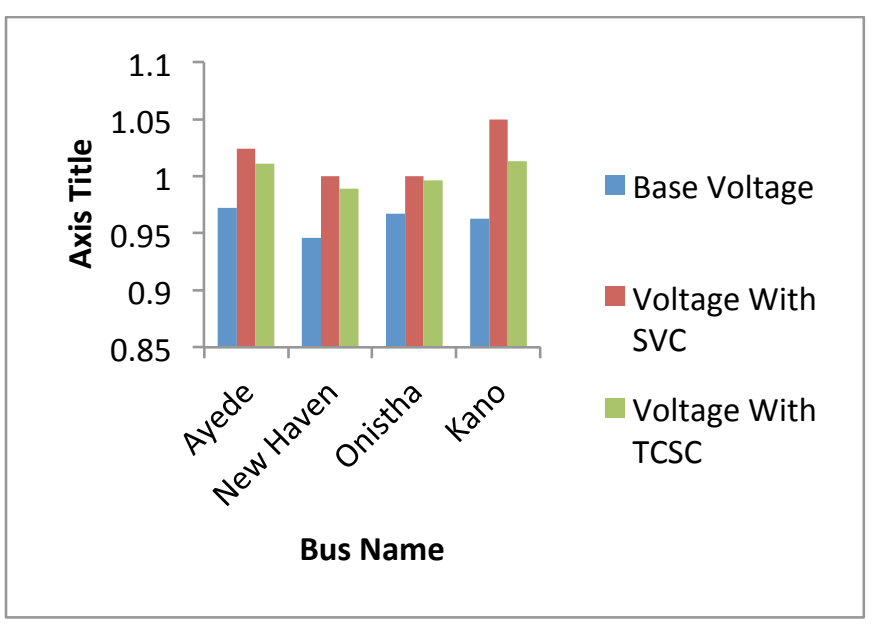

Figure 10: Comparison of Voltage Magnitude in P.U 


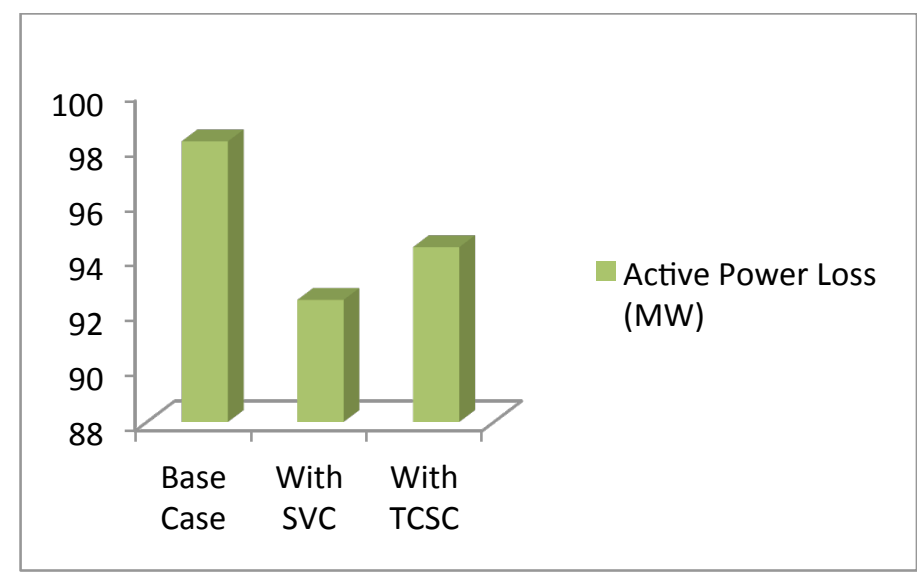

Figure 11: Comparison of Active Power Loss in (MW)

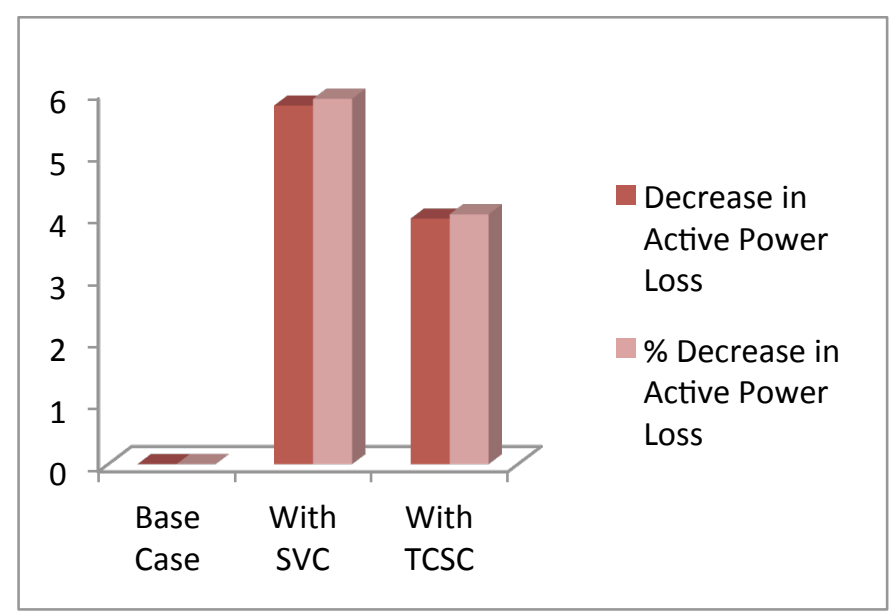

Figure 12: Comparison of Active Power Loss in (MW)

\section{Conclusion}

A comparative analysis of performance evaluation of SVC and TCSC for transmission loss reduction alongside with maintaining voltage magnitude of all the buses within the lower and upper bounds on Nigerian $330 \mathrm{kV}$ grid system Using Self- Adaptive Firefly Algorithm was presented in this paper. The results of the analysis showed that with the system reinforced with SVC, the total system loss reduced from $98.21 \mathrm{MW}$ to $92.44 \mathrm{MW}$ which is about $5.88 \%$ reduction. The reduction in active power loss with the optimal location of TCSCs is $94.259 \mathrm{MW}$ which amount to $4.02 \%$ reduction.

The identified location and parameters of both SVCs and TCSCs using Self-Adaptive Firefly algorithm raised the voltage magnitude of defective buses within acceptable limits of $0.95 \leq V \leq 1.05$ p.u. Applications of SVCs were found to bring appreciable improvement in system's voltage profile in addition to significant reduction in total active power losses compared with what was observed when the system was reinforced with TCSCs.

\section{References}

[1] Dheebika S.K and Kalaivani R (2014): "Enhancement of Voltage Stability by SVC and TCSC Using Genetic Algorithm" International Journal of Innovative Research in Science, Engineering and Technology, Vol.3, Special Issue 3, Pp. 427- 433

[2] Dekhici L., Belkadi K and Borne P (2012): "Firefly Algorithm for Economic Power Dispatching With Pollutants Emission", Informatica Economică, Vol. 16, No 2, Pp. 45- 57

[3] Dixit S., Srivastava L and Agnihotri G., (2004): "Minimization of Power Loss and Voltage Deviation by SVC Placement Using GA", International Journal of Control and Automation, Vol.7, No.6, Pp.95-108

[4] Eiben A and Smit S (2011): "Parameter tuning for configuring and analysing evolutionary algorithms", Swarm and Evolutionary Computation Vol.1, Issue 1, Pp.19-31

[5] Eiben A and Smith J (2003): "Introduction to Evolutionary Computing”, Springer-Verlag, Berlin.

[6] Falcon R., Almeida M and Nayak A (2011): "Fault Identification with binary adaptive firefly in parallel and distributed systems", proceeding of IEEE Congress on Evolutionary Computation, Pp. 13591366

[7] Hingorani N.G and Gyugyi I(2000): "Understanding FACTS: Concepts and technology of Flexible AC Transmission Systems", New York: IEEE Press.

[8] Jumaat S.A, Musirin I, Othman M. M and Mokhlis H (2011): "Transmission Loss Minimization Using SVC Based on Particle Swarm Optimization", IEEE Symposium on Industrial Electronics and Applications (ISIEA2011), September 25-28, Langkawi, Malaysia, Pp.419-424

[9] Mohamed I.R., Khairuddin A and Mustafa M.W (2009): "Optimal allocation of FACTS devices for ATC enhancement using Bees algorithm", World Academy of science Engineering and Technology 3: 313-320

[10] Olabode O. E, Oni D.I and Obanisola O.O (2017): "An Overview of Mathematical Steady-State Modelling of Newton- Raphson Load Flow Equations Incorporating LTCT, Shunt Capacitor and FACTS Devices", International Journal of Advance Research in Science, Engineering and Technology, Vol.4, Issue1, Pp. 3163-3179

[11] Oyedoja K. O (2014): "Modeling and Simulation Study of the Use of Static Var Compensator (SVC) For Voltage Control in Nigeria Transmission Network", International Journal of Engineering and Applied Science, Vol.5, No.05, Pp.44-50

[12] Parate J. V and Sindekar A. S (2012): "Reactive Power Control and Transmission Loss Reduction 
with Realization of SVC and TCSC"

International Journal of Engineering Science and

Technology (IJEST), Vol. 4, No.07, Pp. 3592- 3600

[13] Saraei M., Analouei R and Mansouri P (2015): "Solving of Travelling Salesman Problem using Firefly Algorithm with Greedy Approach", Cumhuriyet University Faculty of Science Science Journal (CSJ), Vol. 36, No: 6, Pp. 266-273

[14] Saravanan M., Mary Raja Slochanal S.,Venkatesh P and Abraham J.P.S (2007): "Application of particle swarm optimization technique for optimal location of FACTS devices considering cost of installation and system loadability", Electrical Power System Research 77: 276-283.

[15] Seifi A.R., Gholami S and Shabanpour A (2010): "Power Flow Study and Comparison of FACTS: Series (SSSC), Shunt (STATCOM) and Shunt-Series (UPFC)", Pacific Journal of Science and Technology, Vol.11, Issue1, Pp.129137

[16] Selvarasu R., S Kalavathi S. M. and Rajan C. C A (2013): "SVC Placement For Voltage Constrained Loss Minimization Using Self- Adaptive Firefly Algorithm", Archives of Electrical Engineering, Vol. 62(4), Pp. 649-661

[17] Shende V.K and Jagtap P.P (2003): "Optimal Location and Sizing of Static Var Compensator (SVC) by Particle Swarm Optimization (PSO) Technique for Voltage Stability Enhancement and Power Loss Minimization", International Journal of Engineering Trends and Technology (IJETT), Vol. 4, Issue6, Pp. 2278-2282

[18] Transmission Company of Nigeria Control Centre, Osogbo, South Western, Nigeria, 2013.

[19] Yang X.S (2010): "Nature-Inspired Meta-Heuristic Algorithms", 2nd ed., Beckington, Luniver Press

[20] Yousif A, Abdullah A.H, Nor S.M., And Abdelaziz A .A (2011): "Scheduling Jobs On Grid Computing Using Firefly Algorithm", Journal of Theoretical and Applied Information Technology, Vol. 33 No.2, Pp. 155-164 